\title{
The elusive diagnosis of sensory neuronopathy
}

\author{
O difícil diagnóstico da neuronopatia sensitiva
}

Hugh J. WILLISON ${ }^{1}$

\author{
1 University of Glasgow, Glasgow, \\ Scotland. \\ Correspondence: \\ Hugh J. Willison; Glasgow \\ Biomedical Research Centre; Room \\ B330, 120 University Place, Glasgow \\ G12 8TA, Scotland; E-mail: hugh. \\ willison@glasgow.ac.uk \\ (iD) https://orcid.org/0000-0002-5997-1683 \\ Conflict of interest: \\ There is no conflict of interest to \\ declare. \\ Received 02 July 2019 \\ Accepted 08 July 2019 \\ (cc) BY
}

I $\mathrm{n}$ this clinical observational study on a large series of consecutive cases of sensory neuronopathy, Alberto Martinez and colleagues ${ }^{1}$ raise awareness of the semiology and causality of diseases affecting the dorsal root ganglia. The article is instructive, tackling the complexities and uncertainties relating to the source of sensory symptoms, and their underlying cause. In doing so, they highlight diagnostic delay and misdiagnosis as recurrent themes in this clinical arena, striking a common chord with many of us working in general neurological practice.

Sensory symptoms manifest in a myriad of tempos, shapes, sites, and sizes, being one of the most common clinical presentations in neurology and arising at a pathological level from every region of the nervous system, from skin to cortex. It is hardly surprising that localizing a symptom complex to a specific anatomical site such as the dorsal root ganglion can be a bewildering exercise. Adding to clinical history and examination uncertainties, the lack of investigational precision in imaging and electrophysiology and the rarity of pathological confirmation in the vast majority of cases add further to the mysteries of disorders arising primarily in the dorsal root ganglion.

Irrefutable evidence for primary degeneration of the dorsal root ganglion cell bodies requires pathological confirmation, which is very rarely conducted in clinical practice, being usually derived from autopsy material. Unsurprisingly, this is most often obtained and therefore best studied in severe, fatal disorders such as paraneoplastic neuronopathy.

Electrophysiological studies on sensory nerves typically interrogate the larger fibers in the distal sensory nerves in the limbs and therefore are not able to discriminate between a local axonopathy, a dying back process due to more proximal injury, or axon loss resulting from neuronal cell body loss. Symmetrical length dependent sensory symptoms and signs are generally regarded as typifying a dying back sensory axonopathy, somewhat excluding a diagnosis of sensory neuronopathy, yet the underlying pathological process may still reside in sub-lethal failure of the sensory neurones to support their longest axons, as occurs in neurodegenerative conditions that at a cell biological level may affect the neuronal cell body.

In clinical practice we rely upon the pattern of symptoms and signs to implicate the dorsal root ganglion - symptoms occur asymmetrically, most predominantly in the legs, often with limb ataxia due to deafferentation, in a multi-segmental pattern rather than a spinal root or peripheral nerve pattern. Electrophysiological changes are restricted to small or absent sensory nerve action potentials with normal motor studies.

Armed with knowledge of these features, the search for underlying cancer or autoimmune disease may yield diagnostic fruit, or draw a blank and be labelled idiopathic, as is the case in the current series where no cause was found in over $50 \%$ of cases.

The odyssey described by Martinez et al. ${ }^{1}$ well describes the pitfalls and failings in identifying sensory neuronopathy as a clinical entity, which is understandable in the context of this complex and interrogation-resistant condition of heterogeneous cases and outcome. Many of us will recall memories of the frustration for both doctor and patient when faced with diagnostic challenges in this arena. Readers will enjoy reading about the author's highlighting of this clinical problem and may reflect on the many cases that slipped through their diagnostic hands, or alternatively those cases on whom they have illuminated the 
diagnosis and cause. When accuracy in diagnosis affects the institution of an important treatment or investigation, a clear understanding of the symptom complex of sensory neuronopathy is particularly important, paraneoplastic associations being the most obvious and critical to identify promptly. Importantly the sensory neuronopathy spectrum remains a practice area where clinical acumen still plays a central role.

\section{References}

1. Martinez ARM, Ribeiro MC, Lima FD, Martins Jr CR, Martins MP,

Nucci A, et al.. Misdiagnosis and diagnostic delay in non-paraneoplastic sensory neuronopathies. Arq Neuropsiquiatr. 2019 Jul;77(7):451-55. https://doi.org/10.1590/0004-282X20190065 